\title{
Synthesis and characterization of amino-terminated polyethylene glycol functionalized mesoporous silica nanoparticles

\author{
Yu Wang ${ }^{1}$, Huameng Gong ${ }^{1}$, Wanxia Wang ${ }^{1}$, Honghao Sun ${ }^{1}$ and Mingxing \\ $\mathrm{Liu}^{1, \mathrm{a}}$
}

${ }^{1}$ College of Biological Engineering and Food, Hubei Provincial Cooperative Innovation Center of Industrial Fermentation, Hubei University of Technology, Wuhan 430068, China

${ }^{a}$ Corresponding author: Imxing@mail.hbut.edu.cn

\begin{abstract}
Keywords: mesoporous silica nanoparticles, functionalization, amino-terminated polyethylene glycol.

Abstract. In this paper, colloidal mesoporous silica nanoparticles functionalized with amino-terminated polyethylene glycol $\left(\mathrm{CMS}-\mathrm{PEG}-\mathrm{NH}_{2}\right)$ were successfully synthesized using silica nanoparticles modified with a carboxy group (CMS-COOH) and amino-terminated polyethylene glycol $\left(\mathrm{H}_{2} \mathrm{~N}-\mathrm{PEG}-\mathrm{NH}_{2}\right)$. Moreover, the particle size and structural properties of CMS-PEG-NH were characterized by transmission electron microscopy (TEM), dynamic light scattering (DLS) and Fourier transform infrared spectroscopy (FT-IR). The obtained results suggested that the CMS-PEG-NH $\mathrm{N}_{2}$ might be a great potential carrier for applying in drug delivery system in the future.
\end{abstract}

\section{Introduction}

Recent research revealed the potential application of mesoporous silica nanoparticles in the fields of biotechnology and nanomedicine, because of their advantages of tremendous surface area, adjustable pore diameter, regular pore structure and facile modification on the internal and external surface [1]. So the mesoporous silica nanoparticles have a potential application in the drug delivery systems. Polyethylene glycol (PEG) can form shield effect through wrapping up the ordered mesoporous materials, which renders them possible to escape the absorption of the reticuloendothelial system (RES) [2] and prolong circulation time in vivo and improve the enhanced permeability and retention (EPR) effect [3]. In our work, we designed a type of mesoporous silica nanoparticles (CMS-PEG- $\mathrm{NH}_{2}$ ) by grafting $\mathrm{H}_{2} \mathrm{~N}-\mathrm{PEG}-\mathrm{NH}_{2}$ onto the surface of $\mathrm{CMS}-\mathrm{COOH}$ via the amide bond linker (Fig. 1a). The terminated amino group could couple with many target moleculars to realize the target delivery. Furthermore, in the further work, we will modify a target moleculars and research the properties of drug loading and controlled release.

\section{Experimental section}

Materials. Tetraethyl orthosilicate (TEOS, 98\%), 3-aminopropyltriethoxysilane (APTES, 98\%), hexadecyl trimethyl ammonium Chloride (CTAC, 97\%), tosyl chloride (99\%), potassium phthalimide $(98 \%)$ and hydrazine hydrate $(98 \%)$ were obtained from Aladdin Chemistry Co. (Shanghai). N-hydroxysuccinimide (NHS, 98\%) and 1-ethyl-3-(3-(dimethylamino)propyl) carbodiimidehydrochloride (EDC. $\mathrm{HCl}, 99 \%)$ were purchased from J\&K Chemical Co. (Beijing). Polyethylene glycol (PEG, Mn=2 kDa), triethanolamine (TEA) and maleic anhydride were purchased from Sinopharm Chemical Reagent Co., Ltd. (China). All the other chemicals are analytical reagent.

Synthesis of carboxy-functionalized colloidal mesoporous silica nanoparticles (CMS-COOH). CMS were synthesized according to a previous study [4]. The introduction of carboxyl group was conducted by dispersing the as-prepared CMS in $30 \mathrm{~mL}$ of ethanol and adjusting the $\mathrm{pH}$ of the 
solution to 10 by ammonium hydroxide. Then, $0.208 \mathrm{~g}$ of APTES-COOH (APTES-COOH was synthesised by APTES and maleic anhydride stiring at $0 \square$ for $15 \mathrm{~min}$ ) was dissolved in $2 \mathrm{~mL}$ of ethanol and added dropwise to the above solution under stiring. The solution was reacted at room temperature for $12 \mathrm{~h}$. Finally, the resulting products were centrifuged, washed with distilled water and ethanol for three times. To remove the template of CTAC, the products were refluxed twice in a solution of $10 \mathrm{~mL}$ of $\mathrm{HCl}(37 \%)$ and $90 \mathrm{~mL}$ of ethanol at $60 \square$ for $2 \mathrm{~h}$, and then the surfactant-removed CMS-COOH were centrifuged, washed with ethanol and distilled water, and redispersed in distilled water $(5 \mathrm{mg} / \mathrm{mL})$ for following use.

Synthesis of amino-terminated polyethylene glycol $\left(\mathrm{H}_{2} \mathrm{~N}-\mathrm{PEG}-\mathrm{NH}_{2}\right) \cdot \mathrm{H}_{2} \mathrm{~N}-\mathrm{PEG}-\mathrm{NH}_{2}$ was synthesized by Gabriel reaction according to previous report [5]. And the synthesis route was presented as Fig. 1(b). Firstly, $10 \mathrm{~g}$ of PEG (5 mmol) and $7.626 \mathrm{~g}$ of tosyl chloride (40 mmol) were dissolved in $100 \mathrm{~mL}$ DCM, followed by adding $8 \mathrm{~mL}$ of triethylamine. The reaction was kept stirring for $24 \mathrm{~h}$ at room temperature. After removing of solvent and precipitating by excess diethyl ether, the tosylated polyethylene glycol (TsO-PEG-OTs) was obtained. Later, $5 \mathrm{~g}$ of dried TsO-PEG-OTs ( $2 \mathrm{mmol}$ ) and $2.408 \mathrm{~g}$ of potassium phthalimide $(13 \mathrm{mmol})$ were dissolved in $80 \mathrm{~mL}$ $\mathrm{DMF}$, reacted at $120 \square$ for $4 \mathrm{~h}$ under nitrogen atmosphere with stirring, and purified as mentioned above. Finally, $4 \mathrm{~g}$ of the phthalimide derivative of PEG (PI-PEG-PI) was dissolved in $70 \mathrm{~mL}$ of ethanol, followed by adding $0.86 \mathrm{~mL}$ of hydrazine hydrate. The reaction lasted for $12 \mathrm{~h}$ at $70 \square$ under stirring and $\mathrm{H}_{2} \mathrm{~N}-\mathrm{PEG}-\mathrm{NH}_{2}$ was obtained after purified as described above.

(a)

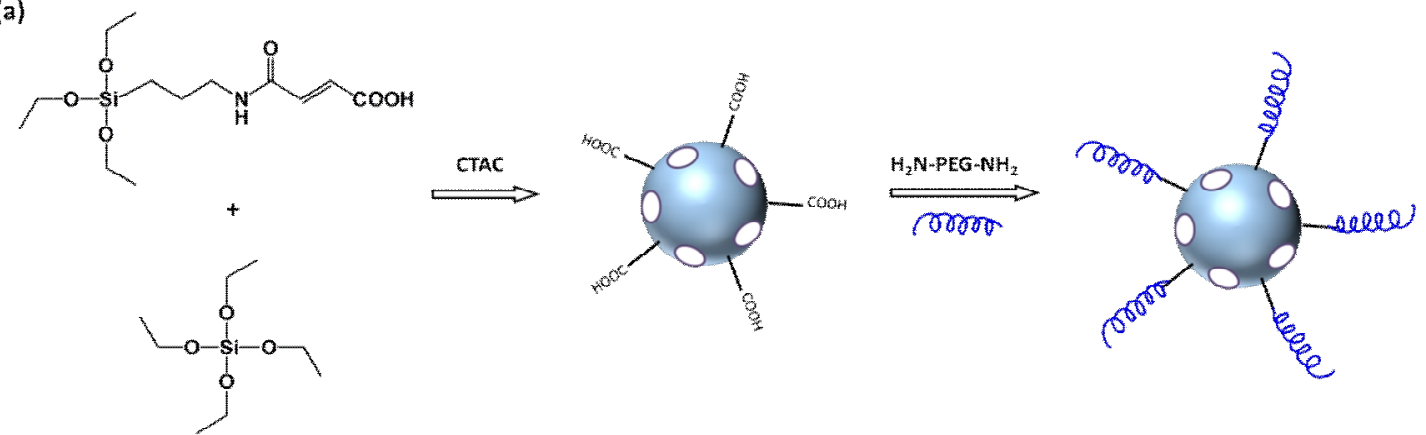

(b)

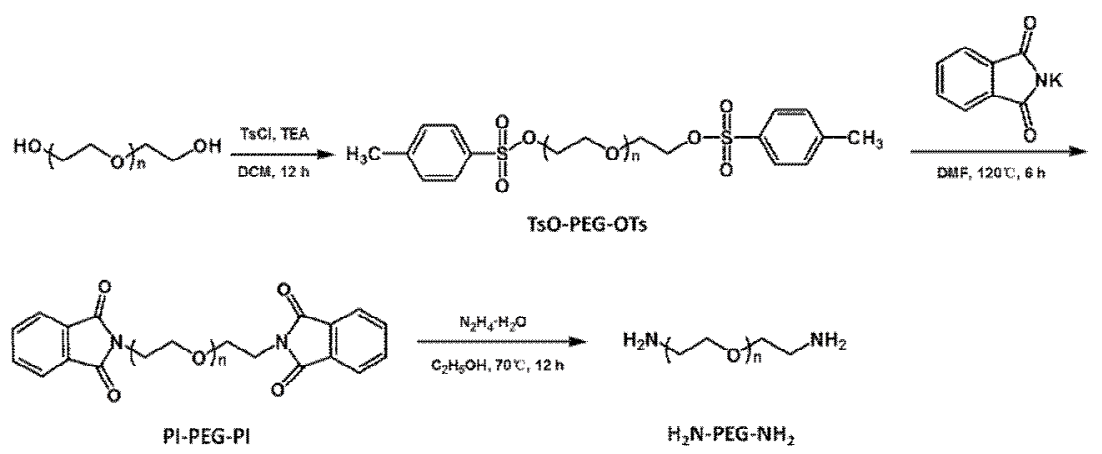

Fig. 1 The synthesis route of (a) mesoporous silica nanoparticles functionalized with amino-terminated polyethylene glycol (CMS-PEG-NH$)$ and (b) amino-terminated polyethylene glycol $\left(\mathrm{H}_{2} \mathrm{~N}-\mathrm{PEG}-\mathrm{NH}_{2}\right)$.

Synthesis of amino-terminated polyethylene glycol functionalized mesoporous silica

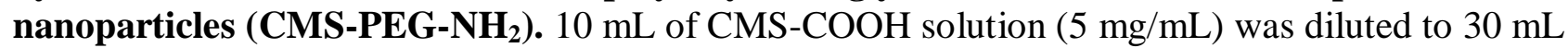
and adjust the $\mathrm{pH}$ to 4.5 approximately using $0.1 \mathrm{M} \mathrm{HCl}$. Then $90 \mathrm{mg}$ of EDC and $60 \mathrm{mg}$ of NHS was added to the above solution and stirred for $1 \mathrm{~h}$ at room temperature. Following, $250 \mathrm{mg}$ of $\mathrm{NH}_{2}$-PEG- $\mathrm{NH}_{2}$ was dissolved in $2 \mathrm{~mL}$ of distilled water and added dropwise to the above solution 
under stiring. The solution was reacted at room temperature for $24 \mathrm{~h}$. Finally, the resulting products were centrifuged, washed with distilled water for several times and redispersed in distilled water to preserve.

Characterization. The zeta potential and size of the nanoparticles were determined with Malvern Zetasizer Nano ZS90. The zeta potential and hydrodynamic diameter were measured at $25 \square$ and $\mathrm{pH}$ 6. Transmission electron microscopy (TEM) images were obtained on a JEOL JEM-2100F transmission electron microscope, and samples for TEM measurements were made by casting one drop of the sample's ethanol solution on carboncoated copper grids. Fourier transform infrared (FT-IR) spectrum was noted on Nicolet Nexus 470. ${ }^{1} \mathrm{H}$ NMR spectra were recorded on a Bruker AM-400 NMR spectrometer in $\mathrm{CDCl}_{3}$.

\section{Results and discussions}

To confirm the successful functionalization of $-\mathrm{COOH}$ on to the surface of PEG, the FT-IR spectra of the Un-CMS and CMS-COOH was shown in Fig. 2. The characteristic peaks showed at 1080 $\mathrm{cm}^{-1}, 800 \mathrm{~cm}^{-1}$ and $960 \mathrm{~cm}^{-1}$ in Fig. 2A could be ascribed to the vibrations of asymmetric stretching $\mathrm{Si}-\mathrm{O}-\mathrm{Si}$, symmetric stretching $\mathrm{Si}-\mathrm{O}-\mathrm{Si}$ and stretching vibrations of $\mathrm{Si}-\mathrm{OH}$ groups, respectively. In Fig. 2B, a new absorption peak appeared at $1701 \mathrm{~cm}^{-1}$ was attributed to the stretching vibration of $\mathrm{C}=\mathrm{O}$. These results demonstrated that the $-\mathrm{COOH}$ had been successfully grafted on the surface of CMS. Besides, a comparison of the FT-IR spectra of the PEG, TsO-PEG-OTs, PI-PEG-PI and $\mathrm{H}_{2} \mathrm{~N}-\mathrm{PEG}-\mathrm{NH}_{2}$ was also shown in Fig. 2. Compared with PEG, a new absorption peak appeared at $1719 \mathrm{~cm}^{-1}$ and $776 \mathrm{~cm}^{-1}$ in Fig. 2D was ascribed to the replace of benzene ring and the in-plane bending vibration of benzene ring, respectively. While the absorption peak appeared at $1597 \mathrm{~cm}^{-1}$ and $662 \mathrm{~cm}^{-1}$ was attributed to the symmetric stretching vibration of $\mathrm{S}=\mathrm{O}$ and stretching vibration of $\mathrm{S}-\mathrm{O}$, respectively. These results demonstrated that the TsO-PEG-OTs had been successfully synthesized. In Fig. 2E, compared with TsO-PEG-OTs, a new absorption peak appeared at 1773 $\mathrm{cm}^{-1}$ and $1712 \mathrm{~cm}^{-1}$ was attributed to the stretching vibration of $\mathrm{C}=\mathrm{O}$ of the cyclic lactam. Besides, the absorption peak at $1597 \mathrm{~cm}^{-1}$ and $662 \mathrm{~cm}^{-1}$ were disappeared. These results confirmed that the sulfonyl group had left and formed the PI-PEG-PI. As shown in Fig. 2F, the absorption peak appeared at $1642 \mathrm{~cm}^{-1}$ was ascribed to the bending vibration of $\mathrm{N}-\mathrm{H}$ of the amino group. And the peak at $1773 \mathrm{~cm}^{-1}$ and $1712 \mathrm{~cm}^{-1}$ were disappeared indicated that the $\mathrm{H}_{2} \mathrm{~N}-\mathrm{PEG}-\mathrm{NH}_{2}$ had been successfully synthesized.

(a)

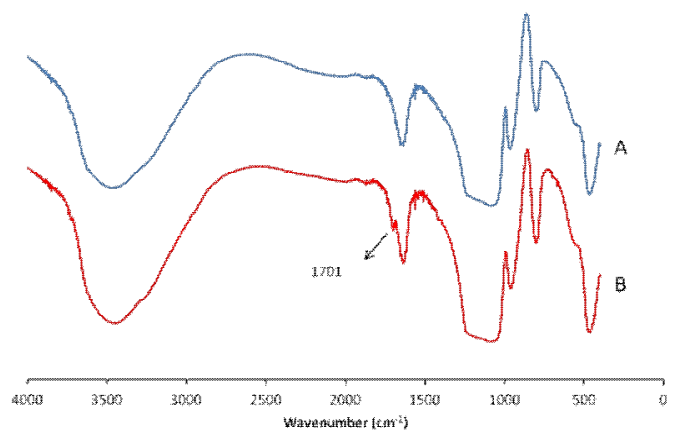

(b)

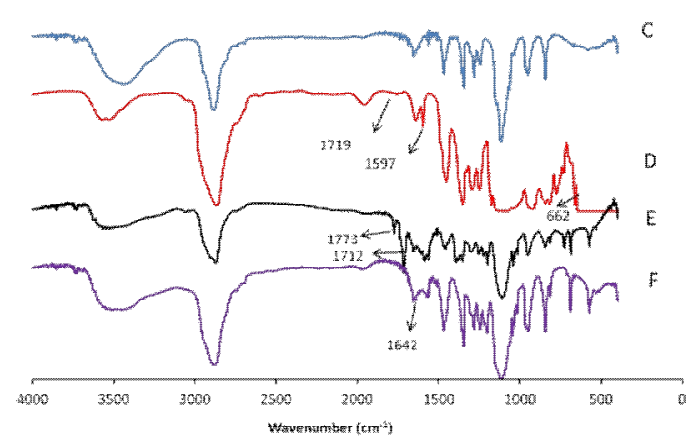

Fig. 2 The FT-IR spectra of Un-CMS (A), CMS-COOH (B), PEG (C), TsO-PEG-OTs (D), PI-PEG-PI (E) and $\mathrm{H}_{2} \mathrm{~N}-\mathrm{PEG}-\mathrm{NH}_{2}(\mathrm{~F})$. 
The structures of $\mathrm{H}_{2} \mathrm{~N}-\mathrm{PEG}-\mathrm{NH}_{2}$ was also determined by ${ }^{1} \mathrm{H}$ NMR analysis (Fig. 3). As displayed in Fig. 3(a), the presence of peak at $\delta 3.57$ corresponded to the protons of repeating unit and $\beta-\mathrm{H}$ of terminal hydroxyl groups of PEG. And the peak at $\delta 3.65$ could be ascribed to the $\alpha-\mathrm{H}$ of terminal hydroxyl groups. In Fig. 3(b), the peak at $\delta 3.57$ which could be ascribed to the protons of repeating unit was remained. While the peak at $\delta 3.65$ was disapeared, and two new peaks apeared at $\delta 3.5$ and $\delta 2.9$ were attributed to the proton of the $\beta-\mathrm{H}$ and $\alpha-\mathrm{H}$ of terminal hydroxyl groups of $\mathrm{H}_{2} \mathrm{~N}-\mathrm{PEG}-\mathrm{NH}_{2}$, respectively. These results suggested that the terminal hydroxyl groups of PEG successfully transformed to the amino group of $\mathrm{H}_{2} \mathrm{~N}-\mathrm{PEG}-\mathrm{NH}_{2}$.

(a)

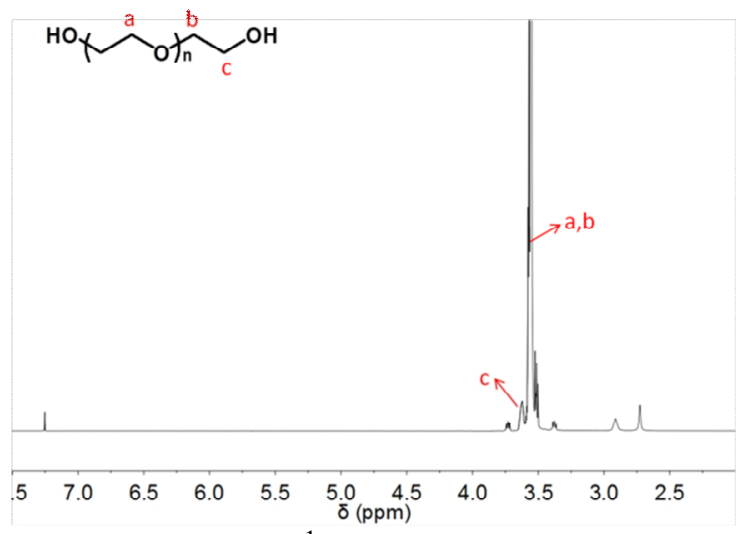

(b)

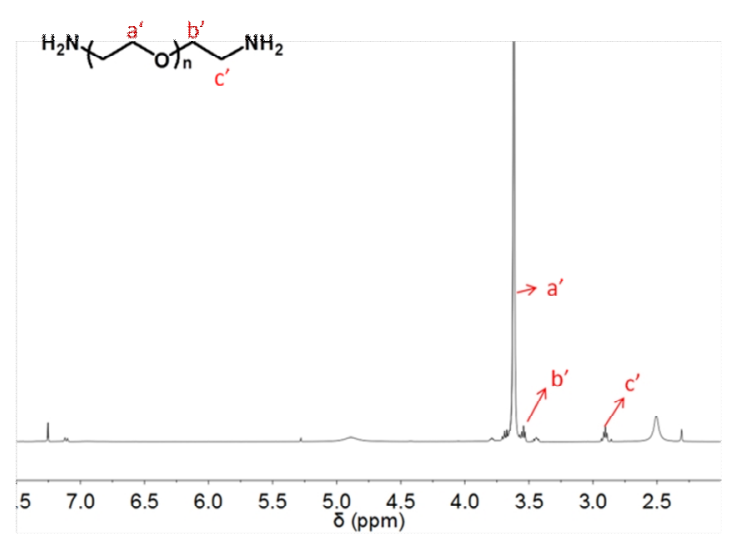

Fig. $3{ }^{1} \mathrm{H}$ NMR spectra of PEG (a) and $\mathrm{H}_{2} \mathrm{~N}-\mathrm{PEG}-\mathrm{NH}_{2}$ (b) in $\mathrm{CDCl}_{3}$.

DLS was used to determine the average particle size and the zeta potential of the nanoparticles. As indicated in Table 1, the average size of the CMS-COOH and CMS-PEG-NH $\mathrm{NH}_{2}$ were $155.5 \mathrm{~nm}$ and $211.3 \mathrm{~nm}$, respectively. Compared with $\mathrm{CMS}-\mathrm{COOH}$, the particle size of CMS-PEG-NH${ }_{2}$ was larger than that of $\mathrm{CMS}-\mathrm{COOH}$, which might be ascribed to the protonation of teminal amino group. Thus leaded to the stretch of PEG chain segments. In addition, the polydispersity index (PDI) of CMS-COOH and CMS-PEG- $\mathrm{NH}_{2}$ were 0.132 and 0.099 , respectively. It indicated that CMS functionalized with amino-terminated PEG was well dispersed in the weak acid solution. Besides, the zeta potential of CMS-COOH and CMS-PEG- $\mathrm{NH}_{2}$ were $-21 \mathrm{mV}$ and $+11 \mathrm{mV}$, respectively. It suggested that amino-terminated PEG was successfully grafted on the surface of CMS due to the charge inversion.

Table 1 DLS analysis of silica nanoparticles

\begin{tabular}{lccc}
\hline Nanoparticles & $\begin{array}{c}\text { Particle size } \\
{[\mathrm{nm}]}\end{array}$ & PDI & $\begin{array}{c}\text { Zeta potential } \\
{[\mathrm{mV}]}\end{array}$ \\
\hline CMS-COOH & 155.5 & 0.132 & -21 \\
CMS-PEG-NH & 211.3 & 0.099 & +11 \\
\hline
\end{tabular}

TEM was carried out to characterize the morphology and pore structure of silica nanoparticles. As presented in Fig. 4(a), the CMS-COOH exhibited a relatively uniform spherical shape with the mean diameter of about $80 \mathrm{~nm}$, and the wormhole arrangement mesoporous was obviously presented. After grafting amino-terminated PEG onto the surface of the CMS, the mesoporous was obscured and the morphology nearly maintained unchanged in Fig. 4(b). In this study, the average size determined by DLS was larger than that of TEM, which could be attributed to the hydration layer when determined by DLS in aqueous condition [6]. 

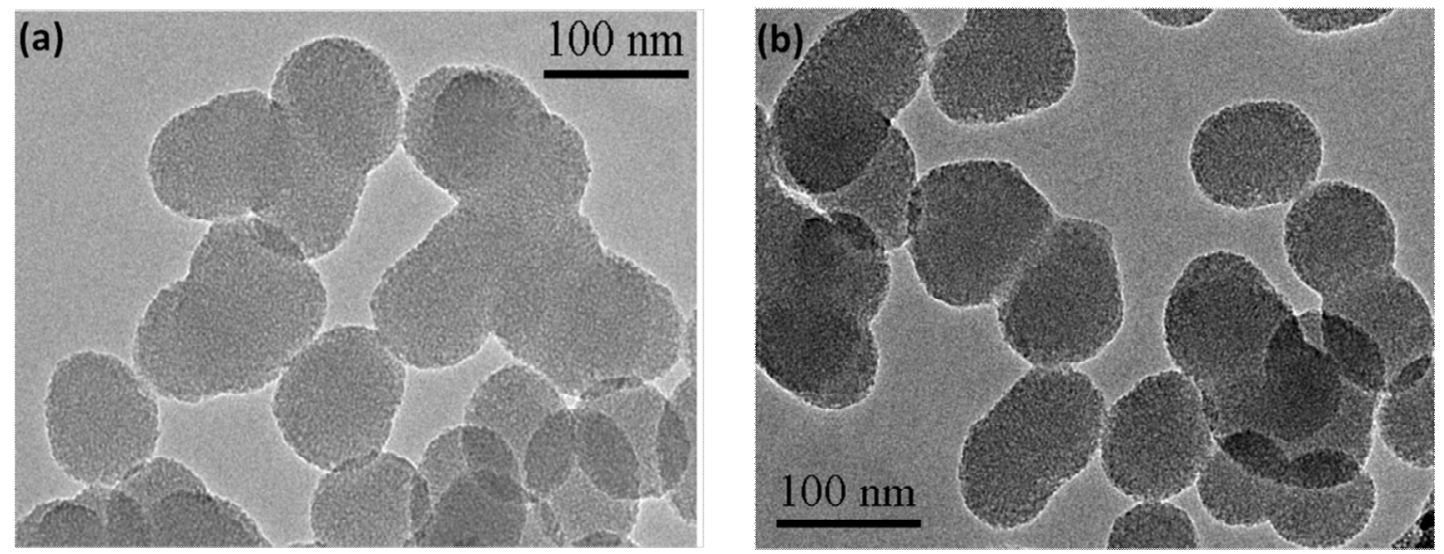

Fig. 4 TEM of CMS-COOH (a) and CMS-PEG-NH 2 (b).

\section{Conclusions}

In this paper, colloidal mesoporous silica nanoparticles functionalized with amino-terminated polyethylene glycol were successfully synthesized using silica nanoparticles modified with a carboxy group and amino-terminated polyethylene glycol. The CMS-PEG- $\mathrm{NH}_{2}$ presented with a relatively uniform spherical shape morphology and favorable dispersibility in weak acid solution. In addition, the terminated amino group could couple with many target moleculars, including folic acid, hyaluronic acid and polypeptide. So It would provide a good platform for the obtained mesoporous silica nanoparticles to applying in target drug delivery system.

\section{Acknowledgements}

This research was financially supported by National Natural Science Foundation of China (No. 81201197).

\section{References}

[1] Z. Li, J.C. Barnes, A. Bosoy, J.F. Stoddart and J.I. Zink: submitted to Chemical Society Reviews (2012)

[2] W. Feng, W. Nie, C.L. He, X.J. Zhou, L. Chen, K.X. Qiu, W.Z. Wang and Z.Q. Yin: submitted to ACS Applied Materials \& Interfaces (2014)

[3] Q.J. He, J.M. Zhang, J.L. Shi, Z.Y. Zhu, L.X. Zhang, W.B. Bu, L.M. Guo and Y. Chen: submitted to Biomaterials (2010)

[4] K. Möller, J. Kobler and T. Bein: submitted to Advanced Functional Materials (2007)

[5] C.L. Shi, X. Guo, Q.Q. Qu, Z.M. Tang, Y. Wang and S.B. Zhou: submitted to Biomaterials (2014)

[6] V. Cauda, C. Argyo and T. Bein: submitted to Journal of Materials Chemistry (2010) 